\title{
Victor Osama, de Francesc Adrià: una propuesta didáctica de análisis de teatro infantil para estudiantes de Grado de Educación Primaria
}

\author{
Moisés SELFA SASTRE \\ Universitat de Lleida \\ Departamento de Didácticas Específicas \\ Facultad de Ciencias de la Educación \\ mselfa@didesp.udl.cat
}

Recibido: mayo 2012

Aceptado: diciembre 2012

\section{RESUMEN}

Victor Osama es una obra de teatro infantil escrita por el dramaturgo valenciano Francesc Adrià y publicada en el año 2010. En este artículo presentamos una propuesta didáctica para el análisis de esta obra teatral que trabajamos con estudiantes de $4^{\circ}$ de Grado de Maestro de Educación Primaria en la materia de Enseñanza y Aprendizaje de Lenguas IV (EyAPL IV) con un triple objetivo: la reflexión sobre el fenómeno del teatro infantil y todo lo que rodea a este género literario, la comprensión lectora del texto y del mensaje que subyace detrás de este y, por último, la definición del concepto de lectura y qué estrategias de comprensión lectora pueden desarrollarse y potenciarse en los estudiantes de 6 a 12 años.

Palabras clave: Víctor Osama, Francesc Adrià, propuesta didáctica, teatro infantil y juvenil, Lectura, competencia lectora.

Victor Osama de Francesc Adrià: an didactic analysis proposal of children's theater for students from Degree in Primary Education

\begin{abstract}
Victor Osama is a children's theatre play written by the Valencian playwright Francesc Adrià, which was published in 2010. In this paper we put forward a didactic proposal based on the analysis of the above mentioned play and we address it to $4^{\text {th }}$ Year Teacher Studies students taking the subject Language Teaching and Learning IV. Such analysis has a threefold objective: reflecting upon the phenomena of playwriting for children and everything that surrounds this literary genre; the reading comprehension of the text and the understanding of the message that underlies in it and, lastly, the definition of the concept of reading and the analysis of the reading comprehension strategies that can be found and that can be promoted among students between 6 and 12 years old.
\end{abstract}

Keywords: Victor Osama, Francesc Adrià, didactic proposal, children's theatre play, Reading, Reading competence. 
Victor Osama du Francesc Adrià: une proposition d'analyse didactique du théâtre des enfants et des jeunes de l'Education Primaire

\section{RESUMÉ}

Victor Osama c'est une pièce de théâtre pour les enfants, écrite par le dramaturge valencien Frances Adriá et publiée à l'an 2010. Dans cet écrit nous présentons une proposée didactique pour son analyse adressée aus étudiants de quatrième degré de professeur en Education Primaire, spécialisés en Enseignement et Apprentissage de Langues IV, avec un tripe objectif: la réflexion sur le phénomène du théâtre d'enfants et pour les enfants et tout ce qui entoure genre littéraire, la compréhension de la lecture du texte et du message qu'il propose, et dernièrement, la définition du concept de lecture et les stratégies de compréhension qui peuvent se développer et devenir intéressantes pour les enfants de six à douze ans.

Mots-clé: Víctor Osama, Francesc Adrià, analyse didactique, théâtre pour enfants et pour la jeunesse, lecture, compétences en lecture.

SUMARIO: 1. Concepto y características del teatro infantil (TI): la lectura y las competencias lectora y literaria. 2. Una propuesta didáctica para el análisis de Víctor Osama, de Francesc Adrià. 2.1. Introducción. 2.2. Objetivos. 2.3. Material. 2.4. Organización de la propuesta y temporalización. 2.5. Procedimiento. 2.5.1. Antes de la lectura. 2.5.2. Durante la lectura. 2.5.3. Después de la lectura. 3. Conclusiones. 4. Bibliografía.

\section{CONCEPTO Y CARACTERÍSTICAS DEL TEATRO INFANTIL (TI): LA LECTURA Y LAS COMPETENCIAS LECTORA Y LITERARIA}

El concepto de teatro infantil (TI en adelante) se refiere, al menos, a dos clases de obras teatrales:

- las obras dramáticas escritas por niños y por adolescentes en las cuales el autor es quien dota al texto de una trama argumental y sentido literario determinado;

- los textos escritos para niños y para adolescentes y que, por lo tanto, tienen presente qué público lector los leerá e interpretará.

Sea cual sea la definición conceptual que se adopte, es evidente que el teatro como texto literario propio y autónomo tiene unas enormes posibilidades de trabajo didáctico en la escuela, en general, y en la Educación Primaria, en particular. En este sentido, este género literario sitúa al estudiante en un doble plano: el del actor y el del espectador. Tanto es así que el discente, actor o espectador, recoge el hecho teatral, lo explota para satisfacer sus necesidades de conocimientos y lo interpreta desde su contexto vital propio.

Desde este punto de vista, el TI abre al niño y al joven las puertas del conocimiento cultural sin ningún tipo de barrera, de la sensibilidad estética, de la reflexión, de la capacidad de emocionarse y de divertirse, de reír y llorar y de, en definitiva, comprender las diferentes visiones y realidades de la vida y del mundo que lo rodea. El TI, además, no sólo está circunscrito al ámbito de la lengua y de la litera- 
tura, sino que la música, la pintura, la danza, el canto y el mimo forman parte de su esencia.

Definido el concepto de TI, podemos referirnos a las características que tiene que tener el texto teatral para poder ser trabajado con sentido en el ámbito de la educación. Entre estas, podemos destacar las siguientes:

- la presencia de un estilo conversacional basado en la interacción directa entre los diferentes personajes del texto;

- la voluntad de usar un lenguaje literario sencillo y asequible a la edad del receptor;

- la aparición de situaciones de acción dinámicas y fáciles de entender;

- la existencia de un planteamiento de la acción bien delimitado que desemboca en un conflicto y una intriga que se mantienen vivos hasta su resolución.

En la escuela, trabajar con textos teatrales que reúnen estos rasgos identificativos se convierte en una actividad de un gran atractivo pedagógico y estético. En palabras de M. Olanya (1999), escuchar, leer, improvisar o montar un teatro afina los sentidos, despierta la imaginación, estimula las valoraciones estéticas, ayuda a la solidaridad con los otros, adiestra la memoria, permite trabajar la lengua de una manera lúdica y facilita la comprensión del texto y de todas y cada una de las palabras que lo componen ayudándose de los signos de puntuación, del tono e intensidad de la voz, de las pausas e incluso del talante de los personajes.

Del mismo modo, el TI favorece el desarrollo de las habilidades intelectuales y de reflexión que tiene que adquirir un estudiante en la etapa de Educación Primaria. Entre estas habilidades ocupa un lugar destacado la lectura y la creación de una sólida competencia lectora en este ciclo de enseñanza.

La competencia lectora consiste, tal como propone la Organización para la Cooperación y el Desarrollo Económico (2001), "en la comprensión y el uso de textos escritos, y en la reflexión personal sobre estos textos con el fin de lograr metas propias, desarrollar el conocimiento y el potencial personal, y participar en la sociedad". Por lo tanto, la lectura de textos literarios, como son los teatrales, es una de las claves para progresar en esta competencia. Y en segundo lugar, aprender a leer con criterio y provecho es un proceso cognitivo que exige una buena implicación personal. La lectura no es simplemente un procedimiento de repetición y reproducción. Es, sobre todo, un aprendizaje que implica un grado alto de comprensión de aquello que se quiere aprender y que se encuentra en un texto.

Para precisar más esta idea nos referiremos a dos cuestiones básicas:

- cuando hablamos de lectura, estamos hablando de un proceso vinculado a la descodificación y a la transformación del conocimiento;

- leer no es una capacidad que se adquiere de una sola vez en un momento determinado de la vida académica de un estudiante. Más bien al con- 
trario, la lectura capacita al discente para interpretar textos cada vez más complejos y de mayor magnitud lingüística y literaria.

Llegados a este punto, podemos preguntarnos cómo se va construyendo esta competencia lectora y qué papel tiene el TI en la escuela para la formación de esta habilidad lingüística.

La competencia lectora se puede empezar a construir bien pronto mediante prácticas teatrales que creen lazos emocionales entre el estudiante y el texto que está leyendo. Si la lectura se refiere a aspectos de la vida cotidiana del estudiante y de su propio mundo, más fácilmente tendrá sentido el texto teatral para este. Así el TI ayuda a representar en la mente del lector un mundo en el cual este es el protagonista principal. El TI, sin duda, facilita el gozo y el aprecio de un tipo de texto que ofrece la oportunidad de aprender a leer para divertirse y para simbolizar.

A pesar de ser imprescindible el hecho de deleitarse con los textos, hay que establecer unas estrategias de lectura para que esta sea funcional y provechosa. La profesora I. Solé (1992) propone tres de estas estrategias que pueden ser perfectamente aplicables al trabajo en el aula con el TI:

- dotar de una finalidad personal la lectura y planificar la mejor manera de leer con provecho;

- inferir, interpretar, integrar la nueva información con el conocimiento previo y comprobar la comprensión durante la lectura;

- elaborar la información, recapitularla, integrarla, sintetizarla y eventualmente ampliarla siempre que la tarea lo requiera.

La competencia lectora no es un ente unitario y hermético. Sin esta no se podrá desarrollar otra competencia que la tradición lingüístico-literaria ha denominado como competencia literaria. En palabras de Dijck $(1972,170)$, la competencia literaria es "la capacidad del hombre para producir e interpretar textos literarios". Leer es, como vemos, dotar de sentido personal un texto hasta el punto de considerarlo como fuente de aprendizaje personal.

Cuando hablamos de competencia literaria y del TI, nos estamos refiriendo a que el discente conozca las características del lenguaje literario teatral. Por eso, podemos decir que el TI contribuye eficazmente, en primer lugar, a la mayor comprensión del texto porque el lector lo tiene que entender bien para poder comunicarlo con intención y sentido. En segundo lugar, acrecienta su expresividad oral: dicción, volumen, entonación y distinción de matices. Y en tercer lugar, enriquece su capacidad de comunicación global puesto que lo ayuda a perder el miedo y a superar inhibiciones, atreverse a levantar la voz cuando el texto teatral lo precise y a imponerse al auditorio ante sus compañeros de representación. Todo esto hace que el estudiante actor haga un esfuerzo de imaginación para recrear escenas en su mente y, así, caracterizar el / los personaje(s) que esté representando. 


\section{UNA PROPUESTA DIDÀCTICA PARA EL ANÁLISIS DE VÍCTOR OSAMA, DE FRANCESC ADRIÀ}

\subsection{Introducción}

Victor Osama es una obra de teatro infantil del dramaturgo valenciano Francesc Adrià. Fue galardonada con el primer premio SGAE (Sociedad General de Autores Españoles) y Fundación Autor de Teatro Infantil en su XI Convocatoria de 2010. El objetivo de estos premios es impulsar y apoyar los textos dramáticos que contribuyan tanto por su originalidad como calidad literaria al enriquecimiento del panorama teatral actual.

La propuesta didáctica de lectura y análisis de esta obra teatral es fruto de la experiencia que se lleva a cabo en los estudios de Grado de Maestro en Educación Primaria de la Facultad de Ciencias de la Educación de la Universidad de Lleida. Concretamente, en la materia de $4^{\circ}$ de Grado de Educación Primaria Enseñanza y Aprendizaje de Lenguas IV (EyAPL IV) que impartimos desde el área de Didáctica de la Lengua y la Literatura, una de las competencias que trabajamos con nuestros estudiantes universitarios es la lectura de textos teatrales que después podrán ser explotados en su futura tarea docente. Lo que pretendemos con el desarrollo de esta competencia es que los estudiantes no sólo conozcan el fenómeno del teatro infantil y los elementos que lo conforman, sino que sobre todo establezcan estrategias de análisis para trabajar correctamente el fenómeno teatral. Junto a esta competencia queremos desarrollar otra: el conocimiento del proceso de la lectura y comprensión del texto teatral, y en general de cualquier tipo de texto, por parte de estudiantes de Educación Primaria. La lectura y el desarrollo de una buena competencia lectora es una de las tareas que los docentes de hoy en día contemplamos como básica en la formación intelectual de nuestros discentes. En este sentido un buen lector es aquél que establece unas correctas estrategias de lectura con la finalidad de ser competente en esta habilidad lingüística.

Visto así el planteamiento didáctico y los objetivos generales de nuestra actuación, a continuación se ofrece de forma detallada el plan de trabajo que se lleva a cabo.

\subsection{Objetivos}

Con esta propuesta didáctica, se busca desarrollar un conjunto de competencias que se desgranarán en los diversos momentos de la guía didáctica y que detallamos a continuación.

\section{Competencia lingüístico-comunicativa}

- Explicar en qué consiste el fenómeno del texto teatral y las características estructurales y de lenguaje que lo definen como tal.

- Comprender el contenido del texto teatral trabajado y el mensaje que subyace detrás de este mensaje. 
- Interpretar el recurso lingüístico-literario de la metáfora como un procedimiento de creación literaria singular.

- Formarse una opinión crítica y razonada sobre la problemática que rodea a los personajes de la obra.

- Fomentar el gusto por la lectura del texto teatral como fuente de enriquecimiento personal.

- Expresar oralmente en qué consiste el proceso de lectura y qué estrategias de comprensión lectora pueden impulsarse en los alumnos de Educación Primaria.

\section{Competencia artística}

- Apreciar lo que significa el fenómeno del teatro como un género de creación personal y como espectáculo dirigido a un público determinado.

- Analizar y valorar la creatividad e imaginación del autor teatral que dibuja unos personajes que actúan en un escenario y tiempo concretos.

\section{Competencia digital}

- Saber utilizar con criterio y provecho las Tecnologías de la Información y la Comunicación, en especial Internet.

\section{Competencia de aprender a aprender}

- Fomentar el trabajo cooperativo como forma de construcción del propio aprendizaje.

- Aplicar estrategias de reflexión para el desarrollo del pensamiento crítico: comparar, asociar, formular hipótesis de trabajo, anticipar, concluir.

- Establecer estrategias de comprensión y visionado de recursos tecnológicos que ayuden a formarse una opinión crítica de lo que es el concepto de lectura y de cómo debe trabajarse ésta en los estudiantes de Educación Primaria.

\subsection{Material}

Para llevar a cabo la propuesta de trabajo, los estudiantes trabajarán con la edición de Víctor Osama publicada por la editorial Anaya (2011). Esta publicación posee, además del texto teatral, una introducción en la que se detalla el argumento de la obra, el escenario en la que se representa, cómo son y actúan los siete personajes de ésta y unas breves páginas finales - tres en concreto- con algunas sugerencias para la lectura e interpretación del texto.

Junto a este material, será necesario el trabajo con un ordenador desde el cual se pueda acceder a los diferentes hipervínculos que detallan más abajo en el apartado de Procedimiento, sobre todo en los de Antes y Después de la lectura. 


\subsection{Organización de la propuesta y temporalización}

La propuesta está diseñada para realizarse, en un principio, en doce sesiones que coincidirían con las tres partes de una guía clásica de lectura. Cada sesión posee una duración de dos horas. Las tres primeras corresponden al momento de Antes de la lectura, las ocho siguientes al Durante la lectura y las dos últimas al Después de la lectura. En cada sesión se quieren trabajar una serie de contenidos que detallamos a continuación. Desde luego, esta temporalización es orientativa y la flexibilidad tiene que ser el denominador común de esta actividad docente.

\subsection{Procedimiento}

\subsubsection{Antes de la lectura}

Una vez que el estudiante ha leído la contraportada que aparece en la edición citada de la obra teatral, rápidamente cae en la cuenta de que este texto aborda, entre otras, dos realidades personales muy serias: el acoso escolar que sufre un niño estudiante y, en segundo lugar, la conciencia que tiene este niño de que es adoptado. En efecto, Víctor Osama es un personaje que acude al taller de inventos y reparaciones del Sr. Bohigues huyendo de unos chicos mayores que lo persiguen. Allí, después de entablar confianza con este señor, le confiesa que él procede de otro país y que es un niño adoptado. Esta circunstancia la sabemos también cuando María, su madre, se lo explica al Sr. Bohigues en la escena 9.

En una primera sesión, los estudiantes deberán reflexionar y formarse una opinión crítica sobre los dos fenómenos explicados, esto es, el del acoso escolar, también llamado bullyng, y el de la adopción de una persona por una familia. Para ello, se propondrá, en primer lugar, el visionado de Libre (http://www.youtube.com/watch? $\mathrm{v}=\mathrm{YOiOgtCnNqU}$ ), un corto de D. Betancor rodado en 2005 de la productora de cine juvenil Gofiator Pictures. En este corto, dos adolescentes, Diego y Marta, sufren acoso continuo en su colegio. La joven, que consigue liberarse de ello, aconseja a Diego que debe seguir luchando.

En segundo lugar, en la literatura actual tenemos un ejemplo muy reciente que también trata el tema del acoso escolar. Se trata de la última novela de M. Casariego, La jauría y la niebla (II Premio Logroño de Novela, 2009), que se desarrolla en el País Vasco y transcurre en un día. En esta obra, uno de los protagonistas, Ander, sufre una grave situación de acoso escolar. Propondremos al estudiante el análisis crítico de un vídeo en el que el autor de esta novela se refiere a este fenómeno del acoso escolar (http://www.youtube.com/watch?v=GbE1HbR0EtM).

En una seguna sesión, los estudiantes abordarán la presencia del tema de la adopción en la literatura infantil. Para ello leerán el artículo publicado en 2011 por A. Ramírez Leiva, "La adopción en la literatura infantil" (http://www.auladelpedagogo.com/2011/02/la-adopcion-en-la-literatura-infantil/, en el que esta autora refiere un conjunto de ocho libros infantiles en los que se aborda este fenómeno. El objetivo es que los estudiantes comprendan hasta qué punto Víc- 
tor Osama se siente un niño adoptado y por qué tiene la necesidad de suplicarle al Sr. Bohigues que sea su abuelo adoptivo.

En una tercera sesión, una vez analizadas dos de las realidades que rodean la vida de Víctor Osama, será necesario que el estudiante trabaje lo que es el fenómeno del teatro y todo lo que envuelve a este espectáculo. Para ello se proporcionará el enlace (http://www.bertamuñoz.es/panorama/indice.html) en el que puede consultarse el texto digital Panorama de los textos teatrales para niños y jóvenes, de B. Muñoz (2006). En concreto se recomendará la lectura del tercer capítulo de esta obra, "Características de las principales colecciones actuales de teatro infantil y juvenil", en el que se definen conceptos claves como qué es el teatro de niños y el teatro para niños y la lectura y representación de obras teatrales infantiles. El objetivo de esta actividad es entender que el texto de Victor Osama posee unos atributos que lo definen como tal frente a otro tipo de género literario: sobre todo que ha sido concebido para ser representado en un escenario muy determinado y que el diálogo de los personajes es lo que caracteriza y define el comportamiento y la identidad de estos.

\subsubsection{Durante la lectura}

Este apartado de trabajo tiene como objetivo prioritario la correcta comprensión del texto y del sentido de este. Francesc Adrià no sólo construye un diálogo en el que los personajes van interaccionando entre ellos y se van mostrando tal como son, sino que, sobre todo, detrás de este hay una mensaje que el lector deberá captar y entender para interpretar correctamente el significado de esta pieza dramática.

A continuación se ofrece una sinopsis de las escenas agrupadas alrededor de un tema o temas centrales. A partir de cada sinopsis propondremos al estudiante qué aspectos de comprensión del texto son los más indicados para captar el mensaje global del texto. Como es lógico, dividiremos el trabajo en varias sesiones.

Primera y segunda sesiones:

Escenas 1-4: En estas cuatro escenas se fragua la amistad entre Víctor Osama y el Sr. Bohigues. Víctor acude al taller de inventos y reparaciones de este personaje donde encuentra un refugio seguro no sólo porque huye de unos chicos mayores que lo están persiguiendo, sino sobre todo porque encuentra en este espacio un lugar para reconstruir su propia vida que está absolutamente rota. Este es el mensaje principal que F. Adrià quiere transmitir a su lector: un niño que quiere dar sentido a su vida a partir de la amistad con un señor mayor al que convertirá en su abuelo.

La primera actividad que se planteará a los estudiantes es identificar en el texto cómo se fragua esta amistad entre Víctor Osama y el Sr. Bohigues. Se trata de una amistad que, al principio, no es nada fácil pues Víctor apunta con un tirachinas al Sr. Bohigues como este personaje le recuerda al principio de la escena 3.

Un segundo aspecto a considerar y trabajar es el de cómo los dos personajes principales de esta obra muestran su identidad personal. Los estudiantes deberán 
prestar atención a qué aspectos del diálogo entre Víctor Osama y el Sr. Bohigues van esclareciendo cómo son por fuera y por dentro estos dos personajes separados por una edad considerable. Al final de la escena 1, el Sr. Bohigues invita a Víctor a visitar de nuevo su taller cuando quiera y que lo recibirá con gusto pero si le dice cuál es su nombre: "Estoy muy agradecido por su ilustre visita, pero difícilmente podré recibirlo en el futuro si no me dice su nombre". Es entonces cuando Víctor le descubre su identidad nominal. Pero no es hasta la escena 4 cuando Víctor Osama abre las puertas de su corazón de par en par al Sr. Bohigues. En este punto de la acción dramática la amistad entre estos dos personajes quedará consolidada para siempre. Dice Víctor: "Yo soy un desdichado afortunado. Mi madre no lo dice así, pero lo pienso yo cuando lo hablamos en la escuela. Los de la zona del mundo de donde yo vengo son desdichados como yo, pero ellos se han quedado allí. Tengo rasgos diferentes. Eso se ve en la cara y en la piel. Soy adoptado. Lo dice mi ma$d r e$ ". El Sr. Bohigues entiende quién es ese niño que ya ha acudido varias veces a su taller buscando algo más que satisfacer su curiosidad por lo que allí se hace y trabaja.

En cuanto al Sr. Bohigues, Víctor sabe su nombre por el cartel que hay colgado en la puerta del taller de este, si bien al principio lo duda dando a entender que las personas que trabajan en un determinado lugar no tiene por qué llamarse como queda escrito en este cartel (escena 3). El oficio del Sr. Bohigues fue, en un principio, el de inventor, pero tuvo que dejar ese oficio por el de reparador de juguetes y objetos porque la gente le pedía inventos imposibles: bolígrafos que hagan los deberes solos y aparatos que hagan estudiar a los niños (escena 4).

Un tercer aspecto que se trabajará en estas dos primeras sesiones es el de los objetos e inventos que Víctor descubrirá en el taller del Sr. Bohigues. Se planteará a los estudiantes que identifiquen el significado real de estos objetos que, en nuestra opinión, son una metáfora de algunas realidades de la vida.

En la escena 3 Víctor Osama llega al taller del Sr. Bohigues y le pide que le arregle un tren. El Sr. Bohigues acepta el encargo pero a cambio de que Osama vaya al librero de la plaza y le consiga un avión que no está en venta pero que le falta para completar su colección de aviones. El avión en sí no tiene más signifícado que el de representar un modelo de avión que se utilizó en la Primera Guerra Mundial, pero el Sr. Bohigues, coleccionista de este tipo de aparatos, quiere que Víctor se lo consiga y que, como niño inocente que es, ablande el corazón del librero que nunca se lo quiso vender. Por tanto, nuestros estudiantes tendrán que considerar y argumentar si el avión en cuestión es el pretexto para simbolizar la ternura de los niños frente a la dureza de los adultos.

Otro de los objetos que Víctor Osama encuentra en el taller del Sr. Bohigues es el de la peonza que escribe. El niño, en la escena 4, insiste al Sr. Bohigues para que le enseñe alguno de sus inventos. Este saca de un cajón blanco este artilugio que lo baila sobre polvos de talco. La peonza dibuja su recorrido sobre unas letras que combinadas ofrecen el mensaje de Bienvenido. El Sr. Bohigues insiste a Víctor 
Osama que cuando la diseñó quiso que sobre todo fuese educada. Por eso trazó el mensaje ya aludido. A partir de este invento y de sus propiedades, el estudiante deberá explicar si la peonza simboliza el camino de la persona humana que se inicia con un Bienvenido a la vida.

Otro de los personajes que aparece por primera vez en esta obra teatral es el de Ricard Flasch. Se trata de un fotógrafo enamorado de la vida que pasa por el taller del Sr. Bohigues para que este último le arregle su maletín de cuero donde guarda las cámaras fotográficas. Flasch le pide al Sr. Bohigues que le ceda dos de sus inventos: una maleta y un libro. Estos dos inventos se los presentará el Sr. Bohigues a Víctor Osama más adelante. Es interesante que el estudiante destaque aquellos aspectos más significativos de cómo el Sr. Bohigues habla a Ricard Flasch de Víctor Osama, sobre todo cuando al final de la escena el Sr. Bohigues se refiere a él como un niño que tiene unos ojos que hablan.

\section{Tercera y cuarta sesiones:}

Escenas 5-7: En estas dos escenas, aparece María, la madre de Víctor Osama. El Sr. Bohigues insiste mucho a Víctor que quiere conocer a su madre. Con el pretexto de que le arregle una muñeca, María visita el taller del Sr. Bohigues. Y cuál va a ser la sorpresa de este personaje cuando descubre que su propio hijo había estado enamorado de María hasta que sus vidas se separaron. Este descubrimiento mueve al Sr. Bohigues a considerarse el abuelo de Víctor pues, de haberse consolidado la relación de su hijo con María, de hecho así lo sería. A partir del diálogo que mantienen estos dos personajes adultos, indicaremos a nuestros estudiantes que describan cómo son los sentimientos de uno y otro hacia la figura de Víctor Osama y qué papel está cobrando este en la vida de ambos. Es necesario destacar que al final de escena 5, el Sr. Bohigues se refiere a Víctor como de un niño que "sabe un montón, y lo que no sabe lo pregunta [...] Es noble". Su madre adoptiva responde que "está endurecido", pero el Sr. Bohigues concluye que "tiene alma".

En la escena 6, el Sr. Bohigues regala un libro a Víctor Osama para su cumpleaños. Este libro es uno de los inventos de este Sr. Lo que lo caracteriza es que sus tapas son de oro. Para leerlo hay que poner la edad con los dedos en la tapa. Víctor no se decide a hacerlo y confiesa que un libro con estas características le produce miedo. Este libro no lo utilizará Víctor hasta la escena 8. El libro es un objeto que a Víctor le produce un goce estético. Como veremos en la actividad que planteamos en el apartado de Después de leer, a Víctor le gusta leer. Para este personaje, la lectura es una fuente de conocimiento y de enriquecimiento personal. A nuestros estudiantes, les propondremos que expliquen qué puede significar el interés que tiene el Sr. Bohigues para que Víctor lea y, además, lea sin prisas y con provecho tal como aparece en esta escena.

En la escena 7 aparece un nuevo personaje de esta pieza teatral: doña Sara, que es la profesora de Víctor Osama. Acude al taller del Sr. Bohigues porque desde que ha vuelto de su baja laboral está observando un comportamiento extraño en su 
alumno. Ese cambio de actitud puede responder a la amistad que el niño está fraguando con el señor Bohigues, que lo convierte en el eje de su vida. La dureza de las intervenciones de doña Sara contrastan con la dulce firmeza de las respuestas del Sr. Bohigues, entre la que destaca una de ellas: "Ese niño puede darnos lecciones a cualquiera de los nosotros". La conversación entre ambos personajes adultos acaba con un portazo de doña Sara que abandona el taller sin haber conseguido su propósito: que el Sr. Bohigues deje de ser un referente vital para Víctor Osama.

La actividad en la que deberán reflexionar los estudiantes es cómo queda caracterizada la exasperada figura de doña Sara para el lector y qué aspectos del Sr. Bohigues lo van convirtiendo progresivamente en el defensor de la identidad de Víctor Osama, un niño por fuera y, quizás, un adulto por dentro.

\section{Quinta sesión:}

Escena 8: En esta escena, Víctor Osama acude decido al taller del Sr. Bohigues a leer el libro de las tapas de oro. Este le insiste en que hay que aprender a leer los libros varias veces. Este libro es el pretexto para que entre en escena otro de los inventos del Sr. Bohigues: el de la maleta. Esta maleta es un invento hermano del libro porque en ella hay papeles en los que hay escritas diferentes palabras. El Sr. Bohigues invita a Víctor a sacar diversos papeles de la maleta y a combinar las palabras que hay escritas en estos: "Usted saca una y yo otra, y las emparejamos". La primera palabra resultante es la combinación de tritura y polvo, es decir, triturapolvo. Esta palabra es utilizada por el Sr. Bohigues cuando se refiere a una "una bicicleta que tiene un embudo y por él entra el polvo, y en la zona de los pedales hay una caja cerrada con rodamientos que trituran el polvo que entra". Otras de las palabras resultantes del invento de la maleta son, entre otras, comeguerras, mecesueños y enroscaideas. A partir de lo explicado, la actividad que trabajarán los alumnos es la identificación del concepto de binomio fantástico (http://www.rmm.cl/index_sub3.php?id_contenido=18630\&id_seccion=106 95\&id_portal=1924) en las palabras creadas por Víctor Osama y el Sr. Bohigues. La expresión de binomio fantástico la acuñó el pedagogo italiano G. Rodari (1985) para referirse a la asociación en una nueva palabra de dos conceptos, en principio, lejanos el uno al otro. Para lograr establecer una relación entre ellos es necesario utilizar todo nuestro ingenio. Para completar esta actividad de trabajo, nuestros estudiantes visitarán la página del Departamento de Orientación del Centro de Profesorado de Andújar (Jaén) en el que aparecen diversas actividades relacionadas con el binomio fantástico (http://orientacionandujar.wordpress.com/2009/01/20/ lectoescritura-binomio-fantastico-en-imagenes/).

En esta escena 8 es cuando por fin Víctor Osama decide llamar por primera vez al Sr. Bohigues abuelo. Mientras están divirtiéndose formando nuevas palabras, Víctor construye una nueva que es la de abuelo. El Sr. Bohigues interrumpe bruscamente el juego y le advierte a Víctor que no le puede llamar abuelo. Víctor le 
replica que su madre le ha contado que podría haber sido su abuelo. Es entonces cuando el Sr. Bohigues, de una vez por todas, decide ser su abuelo para siempre. La reflexión que plantearemos a los estudiantes es que expliquen los diversos motivos por los que el Sr. Bohigues acepta su nueva condición de abuelo de Víctor Osama.

La escena finaliza con otro juego en el que Víctor explica al Sr. Bohigues un juego parecido al del binomio fantástico y que aprendió en su país de origen: la suma de letras y estrellas que veía por los agujeros de los calcetines rotos: "Solo recuerdo que sumaba letras y estrellas por los tomates de los calcetines [...] Encuentras letras en el cielo, si unes estrellas; a veces, sumo estrellas. Tengo localizada una $F$ de doce estrellas. Conozco una $Y$ de siete. He contado una $Q$ de veinticuatro estrellas, la más difícil". El juego de las estrellas es el pretexto para que Víctor, de un modo suave pero inteligente, explique al Sr. Bohigues que el avión de guerra que este anhelaba tener no es más que un símbolo de la capacidad destructora del ser humano: "Lo dice mamá, que el hombre es el animal más destructor de la Tierra”. Si el Sr. Bohigues, aunque no se atreva a decirlo así de claro, acepta ser ya para siempre el abuelo de Víctor, no menos cierto es que este último progresivamente va cambiando la vida de su nuevo abuelo. Una prueba de ello es la profunda visión que Víctor Osama tiene sobre la vida y sobre el ser humano como queda bien reflejado en las palabras que le dirige al Sr. Bohigues sobre el sentido del avión de guerra del que este se había encaprichado. En este sentido, propondremos a los estudiantes que reflexionen sobre cómo es la relación abuelo-nieto que se va fraguando a partir de la sinceridad que preside la relación de ambos personajes. Será necesario destacar que cuando el Sr. Bohigues escucha atentamente la opinión que Víctor Osama tiene sobre el avión de guerra, se funde con éste en un abrazo.

Sexta y séptima sesiones:

Escenas 9-11:

En la escena 9, María, la madre de Víctor, acude de nuevo al taller del Sr. Bohigues para explicarle lo que nunca ha contado a nadie todavía: que ella sacó a Víctor Osama de su país ilegalmente y que falsificó unos papeles en el hospital en el que Víctor estaba ingresado mientras esperaba la llegada de unos medicamentos que necesitaba para el tratamiento que le habían prescrito. El Sr. Bohigues, cuando oye esta explicación, al principio no la acepta, pero a medida que avanza el diálogo parece que no quiere intervenir en esta situación. De hecho cambia de conversación y le pregunta a María por el tratamiento médico que esta está recibiendo. El diálogo entre los dos personajes es sincero, por lo que los estudiantes deberán explicar qué ha llevado a María a contar la gran verdad sobre la verdadera adopción, si así puede llamarse, de Víctor Osama. Parece como si María también considera ya de pleno derecho al Sr. Bohigues como el abuelo de su hijo Víctor. Si no fuese así, no tendría por qué haberle contado cómo consiguió sacar de un modo ilegal a Osama de su país. 
Las escenas 10 y 11 son las que ayudan a entender el mensaje que F. Adrià quiere transmitir con esta obra de teatro. En la escena 10, doña Sara acude acompañada de don Manuel, el director de la escuela a la que acude Víctor, al taller del Sr. Bohigues. Allí también le espera la madre de Víctor, María. Doña Sara quiere saber de dónde procede esa obsesión de Víctor Osama de encabezar todos sus ejercicios escolares con enunciados cuyo denominador común es la voluntad de que el Sr. Bohigues sea su abuelo. El Sr. Artigas, un secretario judicial, entra en escena para resolver el problema: será necesario escuchar a Víctor Osama, un niño "de los que tocan las venitas del corazón”, para que explique su modo de actuar. Víctor accede a la conversación explicando que todo empezó el día en que el Sr. Bohigues lo acogió en su taller con un qué necesitas y a los pocos días le enseñó uno de sus inventos: un libro con tapas de oro que para leerlo tienes que escribir tu edad en sus tapas. En la última página de este libro había escrita una pregunta sobre si el lector de este había encontrado un verdadero amigo. Víctor, al leer esta pregunta, se respondió a sí mismo que sí, que ese amigo era el Sr. Bohigues y qué mal había en que fuese su abuelo.

Desde este punto de vista, se pedirá a los estudiantes que expliquen cómo quedan definidos cada uno de los personajes que intervienen en la obra. Doña Sara ya no pone peros a la relación de Víctor con el Sr. Bohigues, don Manuel es un simple acompañante de la maestra, María respeta la postura que su hijo adopta ante el Sr. Bohigues, el Sr. Artigas es, de momento, un representante de la justicia que cumple con su obligación de escuchar todas a las partes implicadas en el caso que tiene que resolver, el Sr. Bohigues defiende su posición de abuelo adoptivo de Víctor y, finalmente, Víctor Osama relata con tota la naturalidad cuál es su mayor aspiración en el mundo que le ha tocado vivir: tener al Sr. Bohigues como abuelo adoptivo.

En la escena 11 se resuelve todo el entramado. El Sr. Artigas pide a María que cuente la verdad sobre la adopción de Víctor Osama. Esta la explica con toda la frialdad que supone explicar un hecho ilegal. Sin embargo, el Sr. Artigas, símbolo ahora de la justicia moral y no de la justicia legal, resuelve la situación con una solución salomónica: "debo confesar que el problema se ha vuelto de la justicia, de la sordera de las naciones en medio de tanta bomba, de la ceguera de las leyes tan mudas. Dígales que hagan del taller un mundo donde haya inventos y libros y amistad. Dígales que el juzgado no ha podido hacer nada contra los argumentos, ni contra las evidencias. Y digale a Victor que no cambie cuando se haga grande”. El conflicto queda resuelto y, a partir de entonces, Víctor Osama podrá disfrutar de su abuelo de un modo normal, si bien ya lo hacía desde que este le dio cobijo y amistad un día que huía del acoso de unos niños mayores.

La actividad que se trabajará con los alumnos es la explicación de por qué el Sr. Atigas resuelve la situación de este modo y si no será este personaje un símbolo del adulto que todavía conserva un corazón de niño. 
Octava sesión:

Escena final: En esta escena final Víctor Osama disfruta plenamente de la amistad con su nuevo abuelo, el Sr. Bohigues, con el que se divierte con el juego de las palabras que salen del maletín que se miran, a su vez, por el agujero del calcetín. Vuelve a aparecer el fotógrafo Ricard Flasch que realiza unas fotografías a Víctor Osama. Las dos cuestiones finales que proponemos a nuestros alumnos están directamente relacionadas con los dos personajes principales de esta obra:

- ¿ha reconstruido el Sr. Bohigues la vida de un niño roto por dentro?

- Y al revés, ¿ha dado pleno sentido Víctor Osama a la vida del Sr. Bohigues que, de alguna manera, también estaba rota a falta de una verdadera amistad como la que le ofrece Víctor Osama?

\subsubsection{Después de la lectura}

Una vez leída y analizada la obra teatral de Víctor Osama, observamos que una de las habilidades intelectuales por las que siente interés Víctor Osama es la de la lectura de textos. De hecho, ya al final de la escena 1 aparece esta inquietud: "Porque desde que nos mudamos a esta ciudad, paso por delante todos los días cuando voy a la escuela. Y me gusta leer". Esta inquietud es recogida por el otro personaje principal del texto teatral, el Sr. Bohigues, cuando en la escena 8 enseña a Víctor otro de sus inventos: el del libro de las tapas de oro. El Sr. Bohigues quiere que Víctor Osama aprenda a leer y establezca estrategias de lectura válidas: "A los libros hay que respetarlos. Hay que respetar el orden. No ayuda nada precipitar el relato. Debes empezar por el principio. A ver, ¿lees hasta la última línea?" (escena 3). Y más adelante, como si de su maestro se tratase, le aconseja: "Algunos libros hay que leerlos más de una vez y sin ninguna prisa. Sin ansiedad” (escena 3).

Desde este punto de vista, la tarea que proponemos en nuestra guía didáctica es un Webquest que pretende incitar la reflexión sobre cómo establecer estas estrategias de lectura de textos teatrales, pero también de textos en el sentido amplio de este concepto, y qué factores pueden tenerse en cuenta para la correcta implementación de éstas. El contexto de trabajo del cual partimos, como ya hemos dicho, es la asignatura de EyAPL IV en la que se explican y trabajan los procesos y estrategias de lectura para enseñar a leer en lengua castellana a escolares de entre 6 y 12 años. En este sentido, la propuesta de Webquest permite descubrir una determinada información para desarrollar una tarea concreta y determinada: ¿cómo aprenden los estudiantes de Educación Primaria a leer y qué estrategias de lectura pueden ser potenciadas en los discentes de las edades citadas?

El Wequest diseñado, que deberá ser trabajada a lo largo de las dos sesiones finales de esta propuesta didáctica, lo hemos titulado "Lectura, Estrategias y Evaluación de la Comprensión Lectora". Está creado en el entorno virtual de Crear Webquest (http://www.webquest.es/crear-webquest-o-caza-del-tesoro-online) y la dirección electrónica en la cual puede consultarse es la siguiente: http://www.webquest.es/webquest/lectura-estrategias-y-evaluacion-de-la- 
comprension-lectora. Este recurso electrónico posee la siguiente estructura que detallamos y desarrollamos a continuación en cada uno de sus apartados.

\section{Introducción}

Esta herramienta de trabajo está dirigida a estudiantes de $4^{\circ}$ de Grado de Educación Primaria de la Facultad de Ciencias de la Educación de la Universidad de Lérida. La finalidad de su diseño es que estos futuros docentes exploren, analicen y reflexionen a partir de diferentes materiales qué entendemos por los conceptos de lectura y de competencia lectora, en el sentido amplio y objetivo de ambos conceptos, con el objetivo de promover la incorporación, en su futura planificación didáctica, de estrategias de lectura que contribuyan a desarrollar en sus alumnos de Educación Primaria las habilidades necesarias para consolidar esta habilidad lingüística. Del mismo modo se quieren desarrollar en estos estudiantes de Maestro mecanismos y recursos de evaluación que permitan establecer hasta qué punto los objetivos de aprendizaje han sido asimilados y consolidados.

\section{Tarea}

La tarea que hay que desarrollar en este Webquest responde a dos cuestiones muy claras y concretas:

- ¿qué entendemos por los conceptos de lectura y competencia lectora?

- ¿Qué estrategias de lectura podemos impulsar y desarrollar en los alumnos acordes al nivel de enseñanza de éstos?

Proceso

Los pasos a seguir para la realización de la Tarea son los siguientes:

- en primer lugar, el profesor designará grupos de trabajo de cuatro personas.

- Una vez definidos los grupos, cada grupo de trabajo definirá al representante del grupo para la exposición de los resultados obtenidos, el moderador de discusión del grupo, el secretario y el relator que presentará por escrito las conclusiones a las que se han llegado.

- A continuación, hay que localizar y visionar el vídeo "Introducción a la lectura" que corresponde a la intervención de la Dra. Gemma Lluch (Universidad de Valencia) en el II Congreso Leer.es. (http://www.youtube.com/watch? $\mathrm{v}=\mathrm{m} 6 \mathrm{OMVMgwdvw}$ ).

- Una vez analizado el vídeo, en un documento Power Point deberán registrarse el concepto de lectura que tiene esta investigadora y las finalidades que esta habilidad lingüística tiene en la actualidad.

- Posteriormente habrá que acceder al blog http://habilidadlectora.blogspot.com donde aparece la presentación de Feli- 
pe Zayas (Universidad de Valencia). En el mismo Power Point habrá que definir el concepto de competencia lectora según PISA y qué aspectos hay que tener presente para definir concepto.

- A continuación hay que acceder al PDF "La enseñanza de estrategias de comprensión lectora" http://redescolar.ilce.edu.mx/lecturas_BB/diplomado/la_ensenanza_de:com prension_lectora.pdf). En dos diapositivas habrá que definir qué es una estrategia de enseñanza y qué tipo de estas estrategias pueden implementarse en el aula para desarrollar el hábito lector en los estudiantes de Educación Primaria.

- Por último, se visionará un documento en el que Elisa Yuste (Coordinadora del Área de Promoción de la Lectura del Centro Internacional del Libro Infantil y Juvenil) establece criterios para evaluar la lectura en estudiantes de Educación Primaria (http://www.youtube.com/watch?v=oEPk61L_QQY). En cuatro diapositivas distintas se anotarán cuatro de los criterios de evaluación citados.

- Para finalizar, el representante de cada grupo presentará los resultados obtenidos en el Power Point trabajado y a las conclusiones a las que se han llegado sobre los conceptos de lectura y competencia lectora, sobre las estrategias de comprensión lectora trabajadas y qué indicadores de calidad ayudan a evaluar la lectura de un estudiante.

\section{Evaluación}

Los conocimientos que se evalúan en cada grupo de trabajo, hasta un máximo de 10 puntos, están detallados a modo de rúbricas de evaluación en la plantilla que detallamos a continuación:

\begin{tabular}{|c|c|c|c|c|c|}
\hline & Muy bien & Bien & Suficiente & $\begin{array}{c}\text { Requiere } \\
\text { Mejorar }\end{array}$ & $\begin{array}{c}\text { Nota } \\
\text { final }\end{array}$ \\
\hline $\begin{array}{c}\text { Define clara- } \\
\text { mente el con- } \\
\text { cepto de lectu- } \\
\text { ra }\end{array}$ & 2 & 1,25 & 1 & 0 & \\
\hline $\begin{array}{c}\text { Define clara- } \\
\text { mente el con- } \\
\text { cepto de com- } \\
\text { petencia } \\
\text { lectora }\end{array}$ & & & & & \\
\hline
\end{tabular}




\begin{tabular}{|c|l|l|l|l|}
\hline $\begin{array}{c}\text { Define con } \\
\text { claridad qué es } \\
\text { una estrategia } \\
\text { de enseñanza } \\
\text { y qué tipos de } \\
\text { estrategias } \\
\text { favorecen el } \\
\text { hábito lector }\end{array}$ & & & & \\
\hline $\begin{array}{c}\text { Establece con } \\
\text { claridad tres } \\
\text { criterios de } \\
\text { evaluación de } \\
\text { lectura }\end{array}$ & & & & \\
\hline $\begin{array}{c}\text { El contenido } \\
\text { de la exposi- } \\
\text { ción es claro y } \\
\text { conciso }\end{array}$ & & & & \\
\hline
\end{tabular}

\section{Conclusión}

Al finalizar las actividades los discentes se habrán formado un juicio crítico y personal de los conceptos de lectura y de comprensión lectora y de la aplicación didáctica de las estrategias de lectura que hay que impulsar y llevar a cabo en los estudiantes de Educación Primaria para promover su hábito lector.

Desde este punto de vista, conviene destacar los siguientes aspectos que son el resultado del trabajo del Webquest realizado por nuestros estudiantes universitarios:

- En primer lugar, la lectura no es solo una fuente de placer y de entretenimiento, sino que leer es una habilidad lingüística que como personas que somos nos lleva a conversar, a cohesionarnos socialmente, a escribir, a descubrir, a otorgar significados y transformar la información, a formar ciudadanos comprometidos, a gestionar las propias experiencias y, en definitiva, a ser.

- En segundo lugar, la competencia lectora no es una habilidad que se adquiere exclusivamente en los primeros años de la escolarización, sino que la competencia lectora se entiende como un conjunto de conocimientos, habilidades y estrategias que cada individuo construye a lo largo de su vida, en contextos diversos, mediante la interacción con el entorno y con las comunidades donde participa.

- En este sentido, la competencia lectora consiste en comprender y utilizar los textos escritos, reflexionar e implicarse con ellos y alcanzar, de este modo, los objetivos propios de la lectura que conducirán al conocimiento y aprovechamiento del texto en cuestión. 
- Por último, entre las estrategias y motivos de lectura que pueden implementarse en el aula de Educación Primaria para desarrollar una buena competencia lectora, destacaremos las siguientes: contextualizar y explicar por qué se lee un texto y no otro, actualizar los conocimientos previos del texto a leer, ayudar a los estudiantes a formular predicciones e hipótesis y proponer peguntas sobre el escenario, los personajes, los problemas, la acción y la resolución del contenido del texto.

- En cuanto a los criterios de evaluación de la lectura en estudiantes de Educación Primaria, concretaremos tres de básicos: el estudiante debe ser, en primer lugar, capaz de localizar y obtener información explícita en los textos (ideas principales, datos específicos); en segundo lugar, debe realizar inferencias directas, es decir, deducir información o ideas que no se presentan en el texto pero que están claramente implícitas en los mismos; y, en tercer lugar, el lector debe ser capaz de interpretar ideas e información del texto e integrarlas en su conocimiento.

\section{CONCLUSIONES}

La propuesta didáctica que hemos presentado se ha enfocado desde la óptica del aprendizaje significativo y la construcción del propio conocimiento. El docente, en este proceso, es un guía y un apoyo para que el estudiante construya con su trabajo su propio conocimiento. Se han introducido para ello el uso de las Nuevas Tecnologías de la Información y de la Comunicación con el fin de desarrollar, de este modo, la competencia digital y que se pueda trabajar con unos recursos disponibles en el momento inmediato que se precise su uso.

Somos conscientes que cada grupo de estudiantes es un mundo y que unas actividades pueden funcionar en unos pero no en otros. No obstante, y dada nuestra experiencia, podemos afirmar, en primer lugar, que el texto de Víctor Osama resulta, por diversas razones, de especial utilidad para nuestros estudiantes de EyAPL IV ya que les permite trabajar con un texto teatral actual que no dudamos de recomendar para sus futuros discentes de Educación Primaria. Por un lado, favorece la reflexión y la formación de una opinión personal sobre el concepto de TI y las posibilidades de trabajo didáctico que este ofrece. Por otro, el teatro ayuda al estudiante no sólo comprender el sentido literal del hecho dramático en sí mismo, sino que es, además, una representación del mundo interior de los diversos personajes que actúan en él. Desde este punto de vista, calar en el sentido que F. Adrià otorga a su texto teatral contribuye a que los estudiantes se formen una opinión crítica de realidades de la vida que pueden ser, y de hecho son, en algunos casos, inherentes al ser humano: la solitud, la necesidad de compañía y la reconstrucción del propio mundo interior.

En segundo lugar, uno de los objetivos prioritarios de nuestra propuesta didáctica es trabajar cómo se puede desarrollar en los estudiantes de Educación Primaria un interés por la lectura, la comprensión y la interpretación del texto teatral en len- 
gua castellana. De ahí que hayamos dedicado una parte importante de esta guía a la reflexión y análisis de las estrategias de lectura que se tienen que trabajar a lo largo de la Educación Primaria. Si se consiguen aplicar correctamente estas estrategias, se despertará el interés y gusto por la lectura de textos teatrales y de cualquier tipo. El trabajo hecho con el TI en el aula habrá sido, en consecuencia, una ocasión para disfrutar de un tipo de lectura muy gratificante y para comprender uno de los lenguajes más profundos que ha creado el ser humano: el teatro.

\section{BIBLIOGRAFÍA}

ADRIÀ, F. (2010): Víctor Osama, Madrid, Anaya Grupo Autor.

DIJK, T. A (1972): Some aspects of text grammars. A study in Theoretical Linguistics and Poetics, The Hague-Paris, Mouton.

MUÑOZ, B. (2006): Panorama de los textos teatrales para niños y jóvenes, Madrid, ASSITEJ. Disponible en Web: http://www.bertamuñoz.es/panorama/indice.html

OECD (2006). Evaluación de competencias [en línea]. http://www.oecd.org/home/0,3675,en_2649_201185_1_1_1_1_1,00.html [Consulta: 25 abril de 2012]

OLAYA i GALCERAN, M. (1999): "Llibres de teatre a escena!", en Faristol, 13, $5-12$.

RAMÍREZ LEIVA. A. M. (2001): "La adopción en la literatura infantil” [en línea]. http://www.auladelpedagogo.com/2011/02/la-adopcion-en-la-literatura-infantil/ [Consulta: 26 abril de 2012]

RODARI, G. (1985), Gramática de la fantasia. Introducción al arte de inventar historias, Barcelona, Fontanella.

SOLÉ, I. (1992): Estrategias de lectura, Barcelona, Graó. 